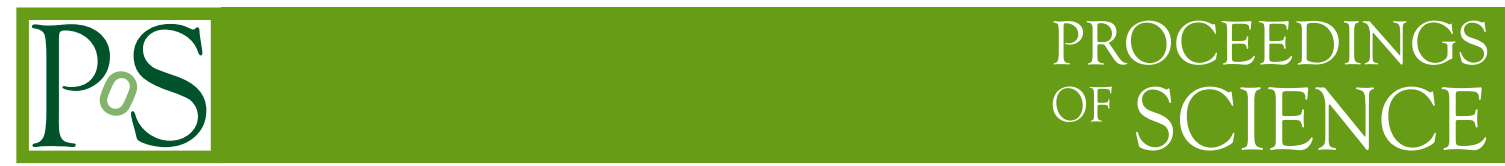

\title{
Measurement of the top quark mass at DØ
}

\author{
Gianluca Petrillo*i \\ University of Rochester \\ E-mail: petrillodfnal.gov
}

The most recent measurements of the mass of the quark top at $\mathrm{D} \varnothing$ are reviewed. The analysis methods include the direct measurement by Matrix Element and Weighting method and the indirect measurement from $t \bar{t}$ production cross section. They have been applied on different experimental signatures, all including at least one electron or muon. Measurements include from 1 to $3.6 \mathrm{fb}^{-1}$ of $\mathrm{D} \emptyset$ data. The most recent combination of mass measurements from $\mathrm{D} \emptyset$ and from $\mathrm{CDF}$ are also quoted.

XVIII International Workshop on Deep-Inelastic Scattering and Related Subjects April 19-23, 2010

Convitto della Calza, Firenze, Italy

\footnotetext{
*Speaker.

$\dagger$ on behalf of the $\mathrm{D} \emptyset$ collaboration
} 


\section{Introduction}

The quark top is the heaviest fundamental particle discovered so far, its mass being almost twice the one of the $Z$ boson and thirty times the next heaviest fermion. It plays a unique role in the Higgs field of the Standard Model, whose coupling strength depends on the mass of the interacting particles. It is also important in the computation of loop corrections, for example for the $W$ boson mass [1].

The top quark is further unique among the quarks in that it decays before it can hadronize [2]. This allows the direct measurement of its mass from its decay products.

The top quark is produced at $\sqrt{s}=1.96 \mathrm{TeV}$ at Tevatron either in $t \bar{t}$ pairs with a cross section of about $8 \mathrm{pb}[3,11]$ or in $t \bar{b}$ with a cross section of about $3 \mathrm{pb}$ [4]. It decays into a $b$ quark and a $W$ boson with a branching fraction larger than $99 \%$. The experimental signature of the $t \bar{t}$ process is therefore determined by the decay channel of the two $W$ bosons.

In the following sections I will describe the Matrix Element and the Weighting methods and the results of their application to $\mathrm{D} \emptyset$ data, an indirect mass measurement from $t \bar{t}$ production cross section and the combination of all DØ and Tevatron measurements.

\section{Matrix element analyses}

The Matrix Element method [5] uses the probability for a detected event to come from a $t \bar{t}$ (signal) process or from a different (background) process.

The probability for an event to come from the signal process is:

$$
P_{\text {sig }}\left(X ; m_{t}, J E S\right) \propto \sum_{\text {flavors }} \int f_{q}\left(x_{1}\right) f_{\bar{q}}\left(x_{2}\right)|\mathscr{M}(\bar{q} q \rightarrow \bar{t} t \rightarrow Y)|^{2} \mathscr{W}(X, Y ; J E S) \mathrm{d} x_{1} \mathrm{~d} x_{2} \mathrm{~d} Y
$$

where $Y$ describes collectively the kinematics of all the particles in the hard scattering final state and $X$ the kinematics of all the objects reconstructed in the detector. The core of this probability is the hard scattering matrix element $\mathscr{M}$ of the signal process $\bar{q} q \rightarrow \bar{t} t$, including $t \bar{t}$ decay. This is wrapped in the probability of having the $q \bar{q}$ initial state with momentum fraction $x_{1}$ and $x_{2}$, product of the parton distribution functions $f\left(x_{1}\right)$ and $f\left(x_{2}\right)$, and by the probability $\mathscr{W}$ for all the fermions in the final state $Y$ to be detected as $X$. The latter has an additional degree of freedom: an absolute jet energy scale shift (JES) which scales all the jet energies by the same factor. Overall, this probability is therefore function of two parameters: the assumed top mass $m_{t}$ included in $\mathscr{M}$ and the JES factor.

The probability $P_{\mathrm{bkgr}}(x ; J E S)$ for an event to come from a background process has the same structure as $P_{\text {sig }}$ (eq. 2.1), except that it's independent of the mass of top quark. We select only the dominant background process for this computation.

On an event-by-event basis, $P_{\text {sig }}$ and $P_{\mathrm{bkgr}}$ are merged together with the signal purity $f$ in one probability of detecting the event, and the probabilities from all the events measured in the selected sample are multiplied together to form a likelihood

$$
L\left(\left\{x_{i}\right\} ; f, m_{t}, J E S\right)=\prod_{i} f P_{\text {sign }}\left(x_{i} ; m_{t}, J E S\right)+(1-f) P_{\mathrm{bkgr}}\left(x_{i} ; J E S\right)
$$


The maximization of $L$ gives the estimators of the signal purity, the residual JES shift and the top mass. These estimators are biased; the actual measurement is extracted from calibration curves which relate the estimators to the unbiased, generated values. These calibration curves are based on our detector simulation.

\subsection{Matrix element analysis on final state with one lepton and jets}

The Matrix Element method has been applied on events which have one electron or one muon reconstructed, plus jets [6]. The $t \bar{t}$ events in this sample have mostly one of the two $W$ bosons from the decay of the top quarks decaying in turn into two quarks and the other into either electron or muon and the respective neutrino. The branching fraction for this process is $\approx 30 \%$.

This channel has an undetected particle in the final state, the neutrino. We estimate its transverse momentum from the detected missing transverse energy $\left(\mathbb{E}_{T}\right)$; the longitudinal momentum can be computed by imposing an invariant mass constraint for the leptons from the $W$ boson: $\left(p_{\ell}^{\mu}+p_{v_{\ell}}^{\mu}\right)^{2}=M_{W}^{2}$.

The main background for this channel is from $W$ boson production associated with jets, which is the process used for the background probability $P_{\mathrm{bkgr}}$; other backgrounds include $Z$ boson and multijet production, where misreconstruction causes a lepton to be lost or faked.

We have applied this analysis on $2.6 \mathrm{fb}^{-1} \mathrm{D} \emptyset$ data, requiring events with an electron or a muon, a minimum amount of $\mathbb{E}_{T}$ and exactly 4 jets. Combining the 312 events with an electron and the 303 with a muon, we obtain a measurement of the mass of top quark $m_{t}=174.5 \pm 1.3($ stat $+\mathrm{JES}) \pm$ 1.4 (syst) $\mathrm{GeV} / \mathrm{c}^{2}$.

Combining this result with the previous measurement on $1 \mathrm{fb}^{-1}$ data, we obtain

$$
m_{t}=173.75 \pm 0.83 \text { (stat) } \pm 1.62(\text { syst }) \mathrm{GeV} / c^{2}
$$

which is compatible with the Standard Model prediction.

\subsection{Top/antitop mass difference}

The $C P T$ invariance theorem prescribes elementary particles and antiparticles to have the same mass. The top is the only quark where this can be measured directly, as the other quarks are always detected in bound states which mask the actual quark mass.

To measure this difference we use the same method described above, with a few differences [7]. Measuring the difference between top and antitop quark $\left(\Delta \equiv m_{t}-m_{\bar{t}}\right)$ relative to their sum greatly reduces the systematic uncertainty. The simulation is done with a customized version of PYTHIA event generator [8] which allows $t$ and $\bar{t}$ quarks to have different masses.

Using $1 \mathrm{fb}^{-1}$ data from events with one electron or muon and four jets, we measure a difference

$$
\Delta=3.8 \pm 3.4(\text { stat }) \pm 1.22 \text { (syst) } \mathrm{GeV} / c^{2}
$$

\subsection{Matrix element analysis on final state with two leptons and jets}

The decay channel of $t \bar{t}$ where both $W$ bosons decay into leptons is extremely pure, but it has two neutrinos in the final state.

We have applied the Matrix Element method in parallel on events with a signature of one electron and one muon [9]. Respective to the analysis with only one lepton, now in our matrix 
element term $\mathscr{M}$ we integrate on all the unmeasured neutrino variables. The main background of this channel is from processes producing a $Z$ boson and jets, when the boson decays in $\tau^{+} \tau^{-}$ and the $\tau$ leptons decay in the leptons pertaining to each signature; additional background comes from $W^{+} W^{-}$production and from $Z$ and multijet production where misreconstruction leads to fake leptons or to an excess of missing transverse energy.

The combination of the measurement on $3.6 \mathrm{fb}^{-1}$ of data for all the two-lepton channels results in

$$
m_{t}=174.8 \pm 3.3(\text { stat }) \pm 2.6(\text { syst }) \mathrm{GeV} / c^{2}
$$

\section{Weighting analyses}

Weighting method is a templates method applied to events with a signature including two leptons and jets.

The $t \bar{t}$ signal events in this channnel have two neutrinos in the final state. Detected quantities don't allow to compute the neutrino momenta: in order to do so, further assumptions are required.

A "weight function" $w_{i}$ is extracted for each event $i$ in the sample. This function always depends on the assumed top quark mass $M_{t}$. Therefore, for each point $w_{i}\left(M_{t}\right)$ the top mass is fixed to $M_{t}$ and used as an additional constraint which contributes to the computation of the momenta of the neutrinos. A charateristic quantity of the weight function, e.g. the $M_{t}$ value $\max _{w, i}$ where the distribution has its peak, is extracted for each event; finally we create the distribution of this quantity from all the events in the sample (fig. 1).

Figure 1: From a simulated sample of $t \bar{t}$ evets with top quark mass $m_{t}=170 \mathrm{GeV} / c^{2}$ : (left) Matrix weight function for one event, as function of assumed top quark mass $M_{t} ;$ (right) distribution of the weight function peaks from the all the events in the sample.
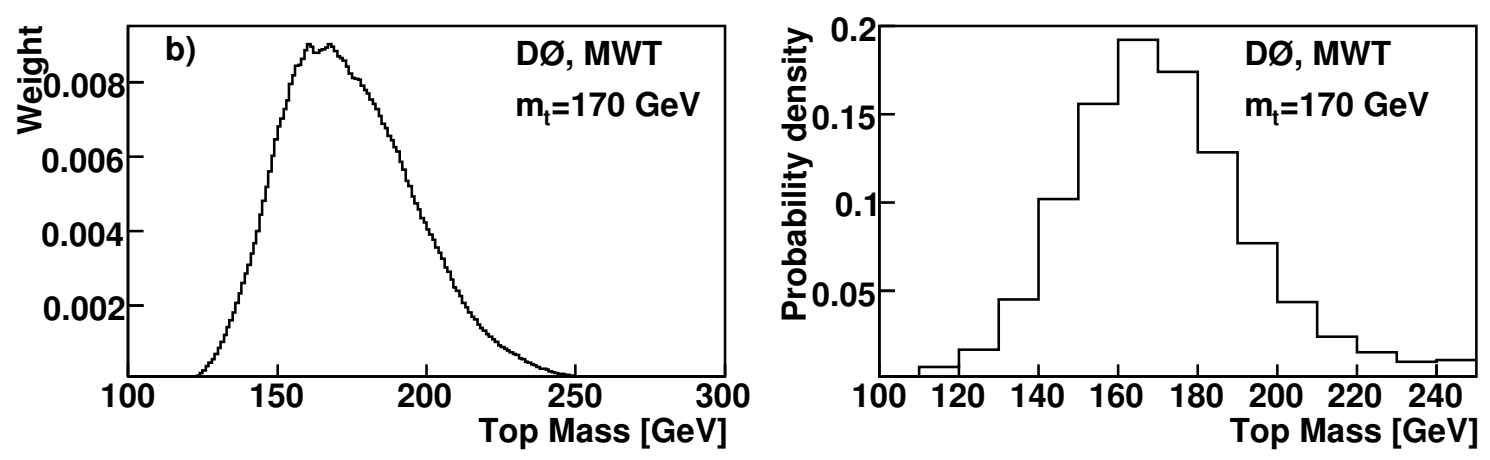

We use simulated samples generated with many different values for the mass of the top quark to extract a set of "templates" distributions. Comparing these distributions with the one extracted from the actual data sample provides an estimator for the mass of top quark. This estimator is biased; a calibration procedure is employed, as in the Matrix Element method, to extract the unbiased measurement.

In this analysis [10] we use two different weights. The first, called Matrix Weight, uses the detected missing transverse momentum together with the assumed mass for top quark to extract the 
neutrino momenta. The weight is then given by

$$
w\left(M_{t}\right)=f\left(x_{1}\right) f\left(x_{2}\right) P\left(E_{\ell}^{*} \mid M_{t}\right) P\left(E_{\bar{\ell}}^{*} \mid M_{t}\right)
$$

with $f$ the parton distribution functions accounting for the probability of the initial state and $P$ the probability to have a lepton with energy equal to the measured one, $E_{\ell \mid \bar{\ell}}^{*}$.

The other weight, called Neutrino Weight, is defined as:

$$
w\left(M_{t}\right)=\exp \left[-\left(\frac{\mathscr{E}_{x}^{\mathrm{obs}}-\mathscr{E}_{x}^{\mathrm{calc}}}{2 \sigma_{x}^{u}}\right)^{2}\right] \exp \left[-\left(\frac{\mathscr{E}_{y}^{\mathrm{obs}}-\mathscr{E}_{y}^{\mathrm{calc}}}{2 \sigma_{y}^{u}}\right)^{2}\right]
$$

which describes the probability of measuring the missing momentum $\vec{E}_{T}^{\text {obs }}$ actually observed, given the one from the neutrinos, $\vec{E}_{T}^{\text {calc }}$, and includes the experimental resolution $\sigma_{x / y}^{u}$ on the missing energy. To constraint the neutrino momenta, we sample their expected pseudorapidity distribution $\left(\eta_{v}, \eta_{\bar{v}}\right)$ to get a set of "subweights" $w^{k}\left(\eta_{v}^{k}, \eta_{\bar{v}}^{k}\right)$. The total weight $w$ is the sum of all of them.

We have applied this method on $1 \mathrm{fb}^{-1} \mathrm{D} \emptyset$ data requiring events with two electron, two muons, one electron and one muon, or one electron or muon and one isolated track (assumed to be a lepton failing tp pass the standard lepton identification criteria). We used the two weight functions presented here independently, obtaining a mass for top quark of $173.2 \pm 4.9$ (stat) \pm 2.0 (syst) $\mathrm{GeV} / \mathrm{c}^{2}$ with the Matrix Weight and $176.2 \pm 4.8$ (stat) \pm 2.1 (syst) $\mathrm{GeV} / c^{2}$ with the Neutrino Weight. Combining these results we find:

$$
m_{t}=174.7 \pm 4.4(\text { stat }) \pm 2.0(\text { syst }) \mathrm{GeV} / c^{2}
$$

\section{Indirect measurements from cross section}

The theoretical prediction of the cross section for the production of top quark pairs depends strongly on the mass of the top quark. The experimental measurement of such a cross section relies on simulation, in which the mass of the top quark is a parameter. Therefore, comparing the predicted and the measured cross section as function of this mass, it is in principle possible to measure the mass of the top quark which best fits both of them.

Many predictions have been published with different level of details included in the computation. We compared our measurement [11] (which is $\sigma(p \bar{p} \rightarrow t \bar{t})=8.18_{-0.87}^{+0.98} \mathrm{pb}$ at $m_{t}=$ $170 \mathrm{GeV} / c^{2}$ on $1 \mathrm{fb}^{-1}$ data), with a pure next-to-leading-order (NLO) prediction [12], one including also soft gluon resummation [13] (NLO+NLL), and two different approximations of next-to-nextto-leading-order cross section $[14,15]$ (NNLO).

The uncertainties on these masses are large; all of them, and especially the most refined ones, are compatible with the current world average (see sec. 5).

\section{DØ and Tevatron combinations}

$\mathrm{D} \emptyset$ has combined the results from the most recent analyses described in this paper together with the older ones from Tevatron Run I [16], obtaining a DØ measurement for top mass of 
Table 1: Mass of top quark for different theoretical predictions of $p \bar{p} \rightarrow t \bar{t}$ cross section. Combined theoretical and experimental uncertainties are shown.

\begin{tabular}{llc}
\hline \hline Prediction by: & type and reference & $m_{t}\left[\mathrm{GeV} / \mathrm{c}^{2}\right]$ \\
\hline Nadolsky et al. & NLO [12] & $165.5_{-5.9}^{+6.1}$ \\
Cacciari et al. & NLO+NLL [13] & $167.5_{-5.6}^{+5.8}$ \\
Moch, Uwer & approx. NNLO [14] & $169.1_{-5.2}^{+5.9}$ \\
Kidonakis, Vogt & approx. NNLO [15] & $168.2_{-5.4}^{+5.9}$ \\
\hline \hline
\end{tabular}

$$
m_{t}=174.2 \pm 0.9(\text { stat }) \pm 1.5(\text { syst }) \mathrm{GeV} / c^{2}
$$

The uncertainties from the different measurements have been combined using the Best Linear Unbiased Estimator [17] (BLUE) technique, which takes into account the correlations of the uncertainties between the different measurements.

$\mathrm{CDF}$ and $\mathrm{D} \varnothing$ have committed into the Tevatron Electroweak Working Group in order to provide what has become the world average for the mass of the top quark, using the same BLUE technique. The last published result [18] includes analyses of up to $3.6 \mathrm{fb}^{-1}$ of data from each experiment:

$$
m_{t}=173.1 \pm 0.6(\text { stat }) \pm 1.1(\text { syst }) \mathrm{GeV} / c^{2}
$$

\section{Acknowledgements}

I'd like to thank the DØ collaboration for the opportunity to present these important results. I also want to thank the people organizing the conference, who had to struggle against an unpredictable event depleting conference rooms and filling their bandwidth, and who did their best to try to accomodate in less than one week the changed needs of the speakers.

\section{References}

[1] G. Degrassi et al., The role of $M(W)$ in precision studies of the standard model, Phys. Lett. B 418, 209 (1998)

[2] The DØ Collaboration, Determination of the width of the top quark, DØ note 6034-CONF (2010)

[3] CDF Collaboration, Combination of CDF top quark pair production cross section measurements with up to $4.6 \mathrm{fb}^{-1}$, CDF note 9913 (2009)

[4] Tevatron Electroweak Working Group, Combination of CDF and DO Measurements of the Single Top Production Cross Section, FERMILAB-TM-2440-E, arXiv:hep-ph/0908.2171 (08/2009)

[5] V.M. Abazov et al., Precise measurement of the top quark mass from lepton+jets events at DO, Phys. Rev. Lett. 101, 182001 (2008)

[6] The DØ Collaboration, Measurement of the Top Quark Mass in the Lepton+Jets Channel Using the Matrix Element Method on $3.6 \mathrm{fb}^{-1}$ of DØ RunII Data, DØ note 5877-CONF (2010) 
[7] V.M. Abazov et al., Direct measurement of the mass difference between top and antitop quarks, Phys. Rev. Lett. 103, 132001 (2009)

[8] T. Sjöstrand et al., PYTHIA 6.4 Physics and Manual, J. High Energy Phys. 0605, 26 (2006)

[9] The DØ Collaboration, Measurement of the Top Quark Mass in the Lepton+Jets Channel Using the Matrix Element Method on $3.6 \mathrm{fb}^{-1}$ of DØ RunII Data, DØ note 5897-CONF (2009)

[10] V.M. Abazov et al., Measurement of the top quark mass in final states with two leptons, Phys. Rev. D 80, 092006 (2009)

[11] V.M. Abazov et al., Combination of ttbar cross section measurements and constraints on the mass of the top quark and its decays into charged Higgs bosons, Phys. Rev. D 80, 071102 (2009)

[12] P.M. Nadolsky et al., Implications of CTEQ global analysis for collider observables", Phys. Rev. D 78, 013004 (2008)

[13] M. Cacciari et al., Updated predictions for the total production cross sections of top and of heavier quark pairs at the Tevatron and at the LHC, J. High Energy Phys. 09, 127 (2008)

[14] S. Moch, P. Uwer, Theoretical status and prospects for top-quark pair production at hadron colliders, Phys. Rev. D 78, 034003 (2008)

[15] N. Kidonakis, R. Vogt", The Theoretical top quark cross section at the Tevatron and the LHC, Phys. Rev. D 78, 074005 (2008)

[16] The DØ Collaboration, Combination of the DØ top quark mass measurements, DØ note 5900-CONF (2009)

[17] A. Valassi, Combining correlated measurements of several different physical quantities, Nucl. Instrum. Meth. A 500, 391 (2003)

[18] The Tevatron Electroweak Working Group, Combination of CDF and DØ Results on the Mass of the Top Quark, CDF note 9717/DØ note 5899 (2009) 